\title{
Factor structure and psychometric properties of the General Health Questionnaire (GHQ-12) among Ghanaian adolescents
}

\author{
Franklin N Glozah ${ }^{1 *}$ and David J Pevalin ${ }^{1}$ \\ ${ }^{1}$ School of Health and Human Sciences, University of Essex, UK
}

*Corresponding Author:

Dr. Franklin Glozah, Department of Psychology and Human Development, Regent University College of Science and Technology, P. O. Box DS 1636, Accra, Ghana.

Email: franklin.glozah@regent.edu.gh 


\begin{abstract}
Purpose: There is little information about the reliability and validity of the 12-item General Health Questionnaire (GHQ-12) in Ghana. This study sought to examine the reliability and factor structure of the GHQ-12 in Ghanaian adolescents.

Methods: High School students (N=770) completed the GHQ-12 and Adolescent Stress Questionnaire (ASQ). Internal consistency, convergent validity and exploratory factor analysis were used.

Results: A two factor structure, each with six items, was extracted. The total GHQ12 had acceptable internal consistency and a generally high correlation with the ASQ subscales.
\end{abstract}

Conclusion: The GHQ-12 can be used in Ghanaian samples, but more research is needed to confirm its factor structure.

\title{
Keywords
}

General Health Questionnaire, Adolescent Stress Questionnaire, adolescents, psychometric properties, common mental illness, Ghana 


\section{INTRODUCTION}

Mental health problems affect between 10 and 20 percent of children and adolescents worldwide but with considerable variation by country (Kieling et al. 2011). Data from the Global School-based Health Survey in Ghana demonstrates that students across the country report experiencing various mental health conditions - felt lonely $(15.2 \%)$, felt so worried $(13.3 \%)$ and felt so sad or hopeless $(37.5 \%)$ (Owusu 2008). A recent systematic review of the psychometric properties of instruments used to screen for major depressive disorder or assess depression symptom severity among African youth found no validation studies or instruments for assessing the mental health of Ghanaian youth (Mutumba, Tomlinson and Tsai 2014). However, mental health and wellbeing among young people have implications for self-esteem, health behaviour, school attendance, educational achievement, social cohesion, future health, and life chances (Olweus 1991).

One widely used instrument for assessing common mental disorder is the 12-item General Health Questionnaire (GHQ-12). The psychometric properties of the GHQ12 have been determined in many English-speaking countries and in other languages (Goldberg 1972). Although it was designed to be a unidimensional instrument, there is no generally agreed upon factor structure of the GHQ-12. Most evidence indicates either a one-factor structure measuring general psychological morbidity (e.g. Pevalin 2000; Ye 2009), a two-factor structure measuring social dysfunction and anxiety/depression (e.g. Montazeri, Harirchi, Shariati et al. 2004; Kalliath, O'Driscoll and Brough 2004; Hankins 2008; Romppel, Braehler, Roth et al. 2013), or a three-factor structure measuring anxiety/depression, social dysfunction, and loss of confidence (e.g. Graetz 1991; French and Tait 2004).

No studies have been conducted in Ghana and among adolescents using the GHQ12. The aim of this study is first, to examine the psychometric properties and explore the factor structure of the GHQ-12 and secondly, to make an initial assessment about the usefulness of using the GHQ-12 in samples of Ghanaian adolescents.

\section{MATERIALS AND METHODS}

\section{Participants and procedure}

The sampling frame consisted of second and third year students in four senior high schools in Accra, Ghana, with an estimated number of 3,000 students. Three classes 
in each of the four schools were randomly selected and any student who was willing and present on the days of questionnaire completion was requested to participate. First-year students were not present at school when the data were being collected. All students present on the days of data collection in all of the 12 selected classes agreed to participate. The response rate was about $97 \%$ due to some students being absent on the days of data collection. The final sample of 770 consisted of 504 males and 266 females with a mean age of 16.9 years. Participants were asked to sign a consent form and those below the age of 18 were given an additional parental consent form for their parent or guardian to sign. As schools in Ghana do not have a specifically nominated ethics committee responsible for research ethics clearance, school authorities including a school counsellor/chaplain confer on issues about confidentiality, anonymity and harm to participants before they give permission for their schools to participate. Ethical approval was obtained from the University of Essex, UK, after school authorities had given permission for their schools to participate in the study.

\section{Measures}

General Health Questionnaire

The GHQ-12 is a well-established, self-administered screening instrument for identifying psychological distress in general population surveys (Goldberg 1972). Participants indicate their agreement or disagreement along a 4-point Likert scale for each item (1- not at all, to 4 - very often). In this study the GHQ-12 total score was the sum of the 12 responses after reversing the six negatively worded items so that the total score ranged from 12 to 48 with high scores indicating higher levels of common mental illness. Tait, French and Hulse (2003) reported Cronbach's $\alpha=0.88$ and Muldoon, Schmid and Downes (2009) reported $\alpha=0.83$ for the GHQ-12 from their sample of adolescents.

\section{Adolescent Stress Questionnaire}

Three subscales - family and home conditions, peer pressure, and teacher interaction - of the Adolescent Stress Questionnaire (ASQ) were used to assess stressful daily life events (Byrne, Davenport and Mazanov 2007). The ASQ is scored on a 5-point Likert type scale with response categories ranging from 1 - never to 5 very often, with higher scores representing more stress perception. Cronbach's $\alpha$ of 
the 19-item ASQ in this study was 0.78 with $\alpha=0.72, \alpha=0.71$, and $\alpha=0.63$ for the home life, peer pressure and teacher interaction subscales respectively.

\section{Statistical Analysis}

Statistical analysis was performed with IBM SPSS 22 software. Exploratory Factor Analysis with a Maximum Likelihood extraction method was used because the primary purpose was to identify the factors underlying the GHQ-12. The KaiserMeyer-Olkin measure of sampling adequacy was 0.81 , above the recommended value of 0.60 (Tabachnick and Fidell 2007). Cronbach's a was used to assess internal consistency. Spearman's correlation coefficient between the GHQ-12 and the ASQ subscales was estimated as an indication of convergent validity.

\section{RESULTS}

\section{Factor structure}

Exploratory Factor Analysis with a direct oblimin rotation method was applied because it was assumed that all extracted factors would be correlated. Only the first two components displayed Eigenvalues greater than 1 indicating that only these first two factors were meaningful - this was also evident in the scree test result. Two items, "Able to enjoy day-to-day activities" and "Feeling reasonably happy" had factor loadings less than 0.30 and cross loaded on both factors in the pattern matrix. As these two items did not contribute to a simple factor structure and also failed to meet the minimum criteria of having a primary factor loading of 0.40 or above (Tabachnick and Fidell 2007), it necessitated that the extraction method specified the extraction of only two factors in subsequent re-analysis. When specifying a two-factor structure, the first factor had an Eigenvalue of 3.29 and explained $27.4 \%$ of the variance whereas the second factor had an Eigen value of 1.53 , explaining $12.7 \%$ of the variance. The two items that initially cross loaded now loaded on one factor each. As the GHQ-12 was originally designed as a unidimensional construct, one-factor structure was also specified and this resulted in an Eigenvalue of 3.22, explaining $26.8 \%$ of the variance. Table 1 shows the factors loadings. In the one-factor structure, there are four items that have loadings lower than 0.4 , but all higher than 0.3. In the two-factor structure, one item on Factor 1 remains below 0.4 . 
Pre-publication version Published doi: 10.2989/17280583.2015.1007867

Table 1: Factor Loadings for the GHQ-12 $(\mathrm{N}=770)$

\begin{tabular}{|c|c|c|c|}
\hline GHQ-12 Item Description & GHQ-12 & $\begin{array}{l}\text { Factor } 1 \\
\text { Social Anxiety }\end{array}$ & $\begin{array}{l}\text { Factor } 2 \\
\text { Social Dysfunction }\end{array}$ \\
\hline Losing confidence & 0.54 & 0.62 & \\
\hline Feeling unhappy and depressed & 0.55 & 0.59 & \\
\hline Thinking of self as worthless & 0.50 & 0.56 & \\
\hline Felt constantly under strain & 0.36 & 0.49 & \\
\hline Couldn't overcome difficulties & 0.42 & 0.48 & \\
\hline Loss of sleep over worry & 0.35 & 0.39 & \\
\hline Able to face problems & 0.52 & & 0.56 \\
\hline Able to enjoy day-to-day activities & 0.51 & & 0.56 \\
\hline Playing a useful part & 0.32 & & 0.50 \\
\hline Able to concentrate & 0.43 & & 0.47 \\
\hline Capable of making decisions & 0.32 & & 0.46 \\
\hline Feeling reasonably happy & 0.51 & & 0.43 \\
\hline
\end{tabular}

\section{Internal Consistency}

Cronbach's a of the GHQ-12 was 0.75 indicating an acceptable level of internal consistency among the 12 items. Ten of the twelve items reached the recommended corrected item-total correlation of 0.30 , indicating reasonable individual item discrimination. The two extracted factors labelled "Social Anxiety" and "Social Dysfunction" each had a moderate Cronbach's a of 0.68. These moderate Cronbach's a may be due to the relatively small number of items in each factor as instruments with a small number of items generate lower alpha values for the same average covariances of and between the items (Cortina 1993).

\section{Convergent Validity}

Results of a Spearman correlation analysis showed statistically significant associations between the GHQ-12 and the ASQ sub-scales, indicating a reasonable level of convergent validity. Table 2 shows the correlation matrix indicating the associations between the composite GHQ-12, the extracted two factor structure and 
the three sub-scales of the ASQ. Overall, these analyses indicated that two distinct factors were underlying adolescents' responses to the GHQ-12 items and that these factors were moderately internally consistent.

Table 2: Spearman Correlation Matrix for the GHQ-12, Two-Factor GHQ and ASQ Sub-scales $(N=770)$

\begin{tabular}{lllllll}
\hline & 1 & 2 & 3 & 4 & 5 & 6 \\
\hline 1. GHQ-12 & -- & & & & \\
2. GHQ-Social Anxiety & $0.79^{* *}$ & -- & & & \\
3. GHQ-Social Dysfunction & $0.84^{* *}$ & $0.36^{* *}$ & -- & & & \\
4. ASQ-Stress of Home Life & $0.35^{* *}$ & $0.35^{* *}$ & $0.22^{* *}$ & -- & & \\
5. ASQ-Stress of Peer Pressure & $0.29^{* *}$ & $0.35^{* *}$ & $0.12^{* *}$ & $0.35^{* *}$ & -- & \\
6. ASQ-Stress of Teacher Interaction & $0.18^{* *}$ & $0.20^{* *}$ & $0.07^{*}$ & $0.29^{* *}$ & $0.28^{* *}$ & \\
M & 35.97 & 5.15 & 13.01 & 21.78 & 11.26 & 6.72 \\
SD & 5.6 & 3.36 & 3.36 & 5.99 & 3.81 & 2.66 \\
Range & $12-48$ & $6-24$ & $6-24$ & $10-46$ & $5-25$ & $4-20$ \\
\hline
\end{tabular}

${ }^{*} p<0.05,{ }^{* *} p<0.01$ (2-tailed)

\section{DISCUSSION}

The purpose of this study was to examine the factor structure and psychometric properties of the GHQ-12 in Ghanaian adolescents and provide empirical evidence with respect to its factor structure, reliability, and association with stressors.

The findings indicate that the GHQ-12 comprises of two factors, each with six items. This two factor structure is similar to the two factor structure found in other adolescent (Li et al 2009) and adult (Kalliath, O'Driscoll and Brough 2004) samples, although each of the factors in these two studies consisted of only four items. In our study, the two items "Felt constantly under strain" and "Loss of sleep over worry" were included in the social anxiety factor while "Playing a useful part" and "Capable of making decisions" were included in the social dysfunction factor. Although half of the GHQ-12 items were reversed scored to get all responses in the same direction, the two factors were extracted strictly in line with how each item was worded (social anxiety-negatively worded and social dysfunction-positively worded), indicating a 
possible response bias towards the items expressing negative mood states (Hankins 2008).

The Cronbach's $\alpha$ of the total GHQ-12 exceeded the minimum threshold and acceptable level suggested by Nunnally and Bernstein (1994), if it were to be viewed as a unidimensional instrument. The slightly lower $\alpha$ values of the social anxiety and social dysfunction factors, is probably due to fewer number of items in each of the factors. This is an indication that the total GHQ-12 could be used in this population as a unidimensional construct because of its acceptable $\alpha$, but interpretation of any measurement should be done cautiously to include the fact that it is has two underlying factors in this population. In comparison, nonetheless, the two-factor structure together explained more variance in common mental disorders $(40.1 \%)$ than the unidimensional GHQ-12 (26.8\%). This suggests that a possible detection of common mental disorders using the unidimensional GHQ-12 will largely be indicative of the detection of both social anxiety and social dysfunction, even though social anxiety will tend to have a greater sensitivity.

Finally, the results showed that the two extracted factors, social anxiety and social dysfunction, had significant associations with the three subscales of the ASQ. The social anxiety factor was particularly strongly associated with the ASQ subscales. This may be due to the response bias towards the social anxiety factor allowing it to explain a higher proportion of the variance in GHQ-12 scores. These moderate associations are indicative of a reasonable convergent validity between the two GHQ factors and ASQ subscales, most probably because of the role, for example, of depression in stressful life events and negative interpersonal relationships (Kendler et al. 1993).

As this study was a cross-sectional survey, retest effects of the GHQ-12 cannot be determined. Nonetheless, given that this is the first study evaluating the GHQ-12 in Ghanaian adolescents, the findings present valuable benchmark data and information for future studies. Also, as the findings were obtained from a sample of second and third year Senior High School students, generalization of the findings to other populations should be done cautiously. 
Taking the GHQ-12 as a unidimensional instrument, as it was originally designed, it has fairly good psychometric properties in terms of internal consistency. As a unidimensional construct, higher scores will indicate a possible detection of common mental disorders, whereas higher scores on each of the two independent factors social anxiety and social dysfunction, will be an indication of a possible detection of social anxiety and social dysfunction respectively. Given that the two-factor structure explains more variance than the unidimensional GHQ-12, it is recommended that the interpretation of the GHQ-12 should be done separately for each of the factors. The results from this study tentatively suggest that the GHQ-12 is a reasonable instrument to detect common mental disorders in Ghanaian adolescents, especially when used as a two-dimensional construct. 
Pre-publication version Published doi: 10.2989/17280583.2015.1007867

\section{References}

Byrne DG, Davenport SC, Mazanov J. 2007. Profiles of Adolescent Stress: The Development of the Adolescent Stress Questionnaire (ASQ). Journal of Adolescence 30: 393-416.

Cortina JM. 1993. What is coefficient alpha? An examination of theory and applications. Journal of Applied Psychology 78: 98-104.

French DJ, Tait RJ. 2004. Measurement invariance in the General Health Questionnaire-12 in young Australian adolescents. European Child and Adolescent Psychiatry 13: 1-7.

Goldberg DP. 1972. The Detection of Psychiatric Illness by Questionnaire. Oxford, Oxford University Press.

Graetz B. 1991. Multidimensional properties of the General Health Questionnaire. Social Psychiatry and Psychiatric Epidemiology 1: 132-138.

Hankins M. 2008. The factor structure of the twelve item General Health Questionnaire (GHQ-12): the result of negative phrasing? Clinical Practice and Epidemiology in Mental Health 4:10.

Kalliath TJ, O'Driscoll MP, Brough P. 2004. A confirmatory factor analysis of the General Health Questionnaire-12. Stress and Health 20(1): 11-20.

Kendler KS, Kessler RC, Neale MC, Heath AC, Eaves LJ. 1993.The prediction of major depression in women: toward an integrated etiologic model. American Journal of Psychiatry 150(8): 1139-1148.

Kieling C, Baker-Henningham H, Belfer M, Conti G, Ertem I, Omigbodun O, Rohde LA, Srinath S, Ulkuer N, Rahman A. 2011. Child and adolescent mental health worldwide: evidence for action. Lancet 378: 1515-1525.

Li WH, Chung JO, Chui MM, Chan PS. 2009. Factorial structure of the Chinese version of the 12-item General Health Questionnaire in adolescents. Journal of Clinical Nursing 18(23):3253-3261.

Montazeri A, Harirchi AM, Shariati M, Garmaroudi G, Ebadi M, Fateh A. 2004. The 12-item General Health Questionnaire (GHQ-12): Translation and Validation Study of the Iranian Version. Health and Quality of Life Outcomes 1: 66.

Muldoon OT, Schmid K, Downes C. 2009. Political Violence and Psychological WellBeing: The Role of Social Identity. Applied Psychology: An International Review 58: 129-145. 
Mutumba M, Tomlinson M, Tsai AC. 2014. Psychometric properties of instruments for assessing depression among African youth: A systematic review. Journal of Child \& Adolescent Mental Health 26:2, 139-156.

Nunnally J, Bernstein L. 1994. Psychometric theory. New York, McGraw-Hill.

Olweus D. 1991. Bully/victim problems among school children: some basic facts and effects of school based intervention programme. In: Pepler D., Rubin K., (eds). The development and treatment of childhood aggression. Hillsdale, Erbaum 411-4187.

Owusu, A. (2008), 'Global School-based Student Health Survey (GSHS): Ghana Report', (Murfreesboro, TN: Middle Tennessee State University, Ghana Education Service, and the World Health Organization).

Pevalin DJ. 2000. 'Multiple Applications of the GHQ-12 in a General Population Sample: An Investigation of Long-term Retest Effects'. Social Psychiatry Psychiatric Epidemiology 35: 508-512.

Romppel M, Braehler E, Roth M, Glaesmer H. 2013. What is the General Health Questionnaire-12 assessing?: Dimensionality and psychometric properties of the General Health Questionnaire-12 in a large scale German population sample. Comprehensive Psychiatry 54(4): 406-413.

Tabachnick BG, Fidell LS. 2007. Using multivariate statistics (5th ed.). Boston, Allyn \& Bacon.

Tait RJ, French DJ, Hulse GK. 2003. Validity and psychometric properties of the general health questionnaire-12 in young Australian adolescents. Australian and New Zealand Journal of Psychiatry 37(3): 374-381.

Ye S. 2009. Factor structure of the General Health Questionnaire (GHQ-12): The role of wording effects. Personality and Individual Differences 46(2): 197-201. 